\title{
Calibration of broadband active acoustic systems using a single standard spherical target
}

\author{
Timothy K. Stanton ${ }^{\mathrm{a})}$ and Dezhang Chu \\ Department of Applied Ocean Physics and Engineering, Woods Hole Oceanographic Institution, Woods \\ Hole, Massachusetts 02543-1053
}

(Received 26 September 2007; revised 27 March 2008; accepted 8 April 2008)

\begin{abstract}
When calibrating a broadband active acoustic system with a single standard target such as a sphere, the inherent resonances associated with the scattering by the sphere pose a significant challenge. In this paper, a method is developed which completely eliminates the source of resonances through isolating and exploiting the echo from the front interface of a sphere. This echo is relatively insensitive to frequency over a wide range of frequencies, lacking resonances, and is relatively insensitive to small changes in material properties and, in the case of spherical shells, shell thickness. The research builds upon the concept of using this echo for calibration in the work of Dragonette et al. [J. Acoust. Soc. Am. 69, 1186-1189 (1981)]. This current work generalizes that of Dragonette by (1) incorporating a pulse compression technique to significantly improve the ability to resolve the echo, and (2) rigorously accounting for the scattering physics of the echo so that the technique is applicable over a wide range of frequencies and material properties of the sphere. The utility of the new approach is illustrated through application to data collected at sea with an air-filled aluminum spherical shell and long broadband chirp signals $(30-105 \mathrm{kHz})$.
\end{abstract}

(C) 2008 Acoustical Society of America. [DOI: 10.1121/1.2917387]

PACS number(s): 43.30.Yj, 43.58.Vb, 43.20.Fn, 43.30.Vh [AJZ] Pages: 128-136

\section{INTRODUCTION}

There is a wide variety of methods to calibrate active acoustic systems. In some cases, it is not essential to know both the source level and receive sensitivity separately and determining the combination of the two, i.e., the system response, would suffice. The standard calibration target has been used commonly as it can determine system response and is frequently convenient to deploy. For example, in the field of fisheries acoustics, solid elastic spheres are considered a standard by which ship-mounted echosounders are calibrated (as reviewed in Simmonds and MacLennan, 2005). In these and other applications, the measured echo from the sphere is normalized by the predicted echo of the sphere (based on known size and material properties) which allows the system response to be calculated.

One major challenge in using targets for calibration involves the resonances of the target [see, for example, thin line in Fig. 1(a)]. The frequencies at which the resonances occur strongly depend on the size and material properties of the target. Minute changes in the resonance frequency, such as due to changes or uncertainties in those physical parameters, can cause significant errors in the calibration at frequencies near the resonance. For narrowband systems, the size and material properties of the sphere can be chosen so that the center frequency of the system occurs between the resonances. This portion of the scattering response is much less sensitive to frequency and material properties and provides a reliable means of calibrating the system. However, for broadband systems, the frequency range may span mul-

\footnotetext{
a) Author to whom correspondence should be addressed. Electronic mail: tstanton@whoi.edu
}

tiple resonances of the sphere and another method must be used. One solution has been suggested which involves use of multiple spheres of various sizes and used one at a time, to connect subbands of near-stationary response of the spheres (Foote et al., 1999; Foote, 2006). Collectively, the spheres could calibrate the system over its entire band. However, for systems of large bandwidth, use of multiple spheres could pose significant logistical constraints. It would be ideal to use a single sphere in a single measurement involving the entire band of the system, without errors associated with the resonances. This paper presents a general approach for the use of a single sphere for the entire frequency band by completely removing the source of the resonances in the time domain.

The resonances are due to interference between the echo from the front interface of the sphere, internally refracted and reflected waves, and various circumferential waves (see, for example, reviews in Hackman, 1993; Marston, 1992; and Numrich and Überall, 1992). These echoes, or "partial waves," can be investigated through use of the exact solution to the sphere in the far field limit and in the backscattering direction. This modal or partial wave series applies to a variety of spherically symmetric profiles of material properties, including solid elastic spheres and liquid- or gas-filled elastic spherical shells. Examples of predictions over a wide range of properties are given in Stanton (1990). Equation (6) in that paper is the modal series solution used in this paper.

Although the modal series solution contains all information regarding the various partial waves, they are inseparable in this current form of the equation. As a result, the equation is normally used to predict the total scattering by the sphere due to all effects, i.e., a "full-wave" prediction, resulting in complex scattering characteristics with many resonances for 

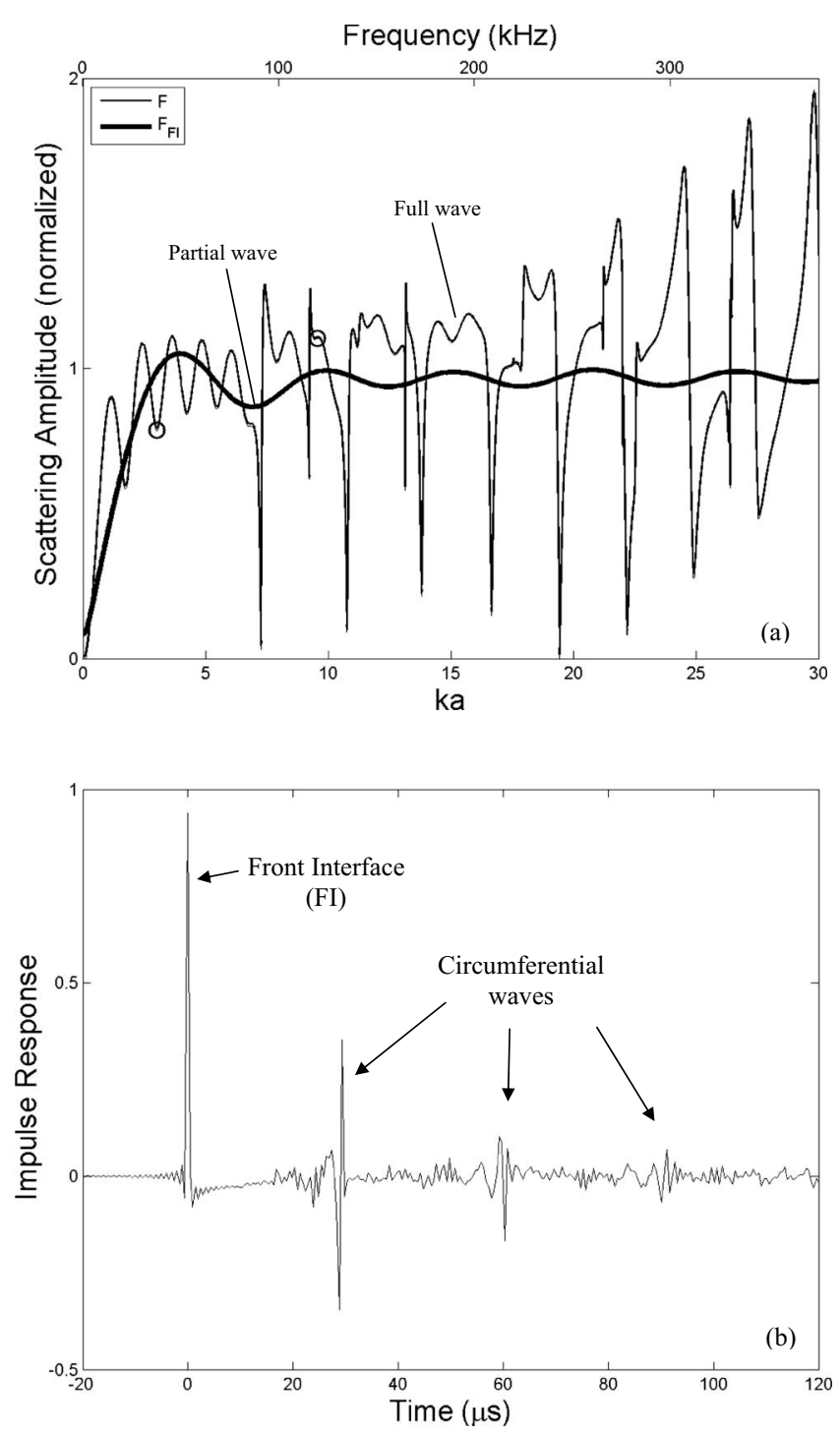

FIG. 1. Acoustic backscattering by a dense solid elastic sphere. (a) The full-wave solution to the sphere (scattering amplitude, $F$; thin curve) which involves a summation of all partial waves and is shown to be strongly dependent upon frequency, containing numerous strong and narrow resonances. The partial-wave solution (scattering amplitude, $F_{F F}$; thick curve) for the echo from the front interface of the sphere which is calculated from the first arrival of the impulse response in Fig. 1(b) and is shown to be weakly dependent upon frequency for higher frequencies. Both solutions for the scattering amplitude are normalized by $a / 2$. In this example, a tungsten carbide sphere of $38.1 \mathrm{~mm}$ diameter was used. The circles correspond to two standard frequencies (38 and $120 \mathrm{kHz}$ ) used in fisheries acoustics. (b) Impulse response of scattering.

values of $k a$ greater than unity [thin line in Fig. 1(a), where $k$ is the acoustic wave number of the surrounding fluid $(=2 \pi / \lambda$, where $\lambda$ is the acoustic wavelength] and $a$ is the radius of the sphere. It is this full-wave analysis that is the basis of the commonly used calibration methods as described in Foote (1982, 2006); Foote et al. (1999, 2007); Feuillade et al. (2002); Simmonds and MacLennan (2005), and others.

The partial waves are inseparable in the modal series solution principally due to the fact that the equation is in the frequency domain. In this "continuous wave" formulation, echoes arriving at different times are not resolved. The waves can be resolved through calculating the impulse response of the sphere, which can be determined by calculating the inverse Fourier transform of the modal series solution (see, for example, Sun et al., 1991; Kaduchak et al., 1995). The resulting time-domain expression is composed of the partial waves arriving at different times [Fig. 1(b)]. The first echo to arrive is from the front interface ("FI"), while all other echoes, such as due to internally refracted and reflected waves and circumferential waves, arrive at later times.

Through various methods, and as given below, the scattering by the front interface has been shown to vary weakly with frequency (at fixed radius) over a wide range of frequencies for $k a>1$ [thick line in Fig. 1(a)] (see, for example, Marston, 1992). The sum of all of the remaining partial waves is strongly dependent upon frequency. The predictions in Fig. 1(a) show that in the limit of high frequencies (high $k a$ at fixed $a$ ), the echo from the front interface is independent of frequency. This has also been shown analytically:

$$
F_{\mathrm{FI}} \simeq \frac{1}{2} a R e^{-i 2 k a}, \quad k a \gg 1,
$$

where $R=(g h-1) /(g h+1)$

$$
g \equiv \rho_{2} / \rho_{1} ; \quad h \equiv c_{2} / c_{1} .
$$

Here, $F_{\mathrm{FI}}$ is the scattering amplitude in the backscattering direction of the echo from the front interface; $R$ is the planewave, plane-interface reflection coefficient for the front interface; $i=\sqrt{-1}, g$ and $h$ are the density and sound speed contrasts, respectively, of the sphere; and $\rho$ and $c$ are density and sound speed, respectively. Medium " 1 " is the surrounding water and medium " 2 " is the sphere. Here, the upper case notation for $F_{\mathrm{FI}}$ and other terms throughout this paper refers to the frequency domain while the lower case notation (for example, $f_{\mathrm{FI}}$ ) refers to the time domain. Also, the partial wave target strength $T S_{\mathrm{PW}}$ for the echo from the front interface, as defined by Chu and Stanton (1998), is $T S_{\mathrm{PW}}$ $=10 \log \left|F_{\mathrm{FI}}\right|^{2}$.

Given the fact that the echo from the front interface is relatively insensitive to frequency, it is desirable to use this echo from a standard target for calibrating a system. This fact was recognized by Dragonette et al. (1981) who used the echo in a partial-wave analysis to calibrate a broadband system. In that study, they achieved the resolution needed through use of a short high frequency signal. The analysis showed excellent agreement between the predicted and measured form function (which is related to scattering amplitude).

Part of the success of their results was attributed to the fact that the experiments were at very high frequenciesvalues of $k a$ ranging from 14 to 70 . In that region, the echo from the interface can be assumed, to first order, to be independent of frequency, as shown in Eq. (1). The analysis also benefited by the use of a dense solid calibration sphere, as the amplitude of the echo from the front interface was assumed to be due to a rigid sphere [i.e., $R=1$ in Eq. (1)]. Also, since the system used very short pulses, then the first echo could be resolved simply through analyzing the raw, unprocessed, echo. The pulses were made short through limiting the transmit signal to just a small number of cycles of high frequency sound. The assumptions that the first echo is independent of frequency and due to a rigid sphere, as well as the 
use of the raw echo, limits the calibration with this approach to high values of $k a$, dense solid spheres, and short transmit signals. Because of these limitations, the calibration method is not practical for calibration spheres of less dense materials and systems that operate at lower frequencies and/or with long transmission signals.

In this paper, the partial-wave analysis of Dragonette et al. (1981) is generalized to cover a much wider range of frequencies, transmit signals with long durations, and calibration spheres with a wide range of materials. The generalization involves a combination of applying a signal processing technique to the echo and accounting rigorously for the scattering physics associated with the first echo:

(1) Signal processing. Rather than using the raw echo, whose range resolution is limited by the duration of the transmitted signal, the broadband echo is processed through cross correlating it with a replica signal. This signal processing significantly reduces the duration of the echo (similar to matched filter processing) and the duration of the compressed echo is now related to the inverse bandwidth of the system, which can be much shorter than the duration of the original raw signal.

(2) Scattering physics. Rather than assuming that the echo from the front interface is independent of frequency and due to a rigid sphere, the characteristics of the echo are exactly calculated. This is obtained through calculating the impulse response (time domain) of the exact solution (frequency domain) of the scattering function and temporally isolating the first echo in the impulse response for analysis. The exact solution can be applied to a wide range of material properties of spheres including solid elastic (any material) and elastic spherical shell (any material and shell thickness) filled with gas or liquid. Furthermore, the equation is exact for all frequencies.

The paper is organized as follows: In Sec. II, the raw, unprocessed echo voltage received in an experiment due to a target is expressed in the frequency domain in terms of the applied voltage, system response, and scattering amplitude of the target. In Sec. III, pulse compression processing is incorporated into those equations, resulting in an equation for system response in terms of the pulse-compressed signals associated with the calibration sphere. In Sec. IV, the results from Sec. III are applied to data collected in an at-sea calibration where the duration of the raw broadband signal corresponds to a distance of more than three times the diameter of the calibration sphere. Finally, in Sec. V, recommendations are made for the design of spheres for broadband calibrations. Here, a sensitivity analysis is conducted demonstrating how use of the echo from the front interface is relatively insensitive to small changes in material properties and thickness of shell. Also, design curves are given for recommended diameter for a range of bandwidths and several types of spheres.

\section{GENERAL EQUATIONS FOR SYSTEM RESPONSE}

The voltage, $V_{r}(\omega)$, of the received echo due to the scattering by a target located arbitrarily in the acoustic beam can be written as:

$$
\underbrace{V_{r}(\omega)}_{\begin{array}{c}
\text { received } \\
\text { voltage }
\end{array}}=\underbrace{V_{t}(\omega)}_{\begin{array}{c}
\text { applied } \\
\text { voltage }
\end{array}} \underbrace{H_{0}(\omega) B_{r}(\omega) B_{t}(\omega)}_{\begin{array}{c}
\text { system response } \\
H(\omega)
\end{array}} \underbrace{\frac{r_{\text {ref }}}{r^{2}} e^{i(2 \omega / c) r} e^{-2 \alpha(\omega) r} F_{\mathrm{bs}}(\omega)}_{\begin{array}{c}
\text { transmission loss } \\
L(\omega)
\end{array}}, \underbrace{r^{2}}_{\begin{array}{c}
\text { scatterer } \\
\text { response }
\end{array}}
$$

where $\omega$ is the angular frequency, $V_{t}(\omega)$ is the voltage applied to the transmitter transducer, $H_{0}(\omega)$ is the system response of the combination of transmitter and receiver transducers evaluated in the center of the main lobe of the beam pattern (i.e., the product of the voltage-to-pressure and pressure-to-voltage ratios of the transducers), $B_{r}(\omega)$ and $B_{t}(\omega)$ are the beam patterns (normalized to unity in the center of each main lobe) of the receiver and transmitter transducers, respectively, $r_{\text {ref }}$ is the reference distance at which the voltage-to-pressure ratio is measured for the transmitter transducer, $r$ is the distance between the transceiver and the target, $c$ and $\alpha(\omega)$ are the sound speed and absorption coefficient, respectively, of the water, and $F_{\mathrm{bs}}(\omega)$ is the backscattering amplitude of the target.

This equation is written in general form and can be applied to both the calibration target as well as targets of interest in a field application. Furthermore, it can be used for either the commonly used full-wave calibration in which the exact modal series solution is used to include all of the scattering components or the partial-wave calibration, which is the focus of this paper, in which a subset of the scattering components, such as only the echo from the front interface, is used. For the "steady state" solution in which all partial waves contribute to $F_{\mathrm{bs}}(\omega)$, then the traditional definition of target strength applies: $T S=10 \log \left|F_{\mathrm{bs}}(\omega)\right|^{2}$. However, if this equation is applied to partial waves, such as in subsequent sections, the partial-wave target strength applies, as discussed earlier as well as in detail in Chu and Stanton (1998).

For the calibration, the above equation is written in more compact form, with the superscript "(cal)" assigned to quantities corresponding to the calibration measurement:

$$
V_{r}^{(\mathrm{cal})}(\omega)=V_{t}^{(\mathrm{cal})}(\omega) H(\omega) L^{(\mathrm{cal})}(\omega) F_{\mathrm{bs}}^{(\mathrm{cal})}(\omega) .
$$

At this point, Eq. (3) is one equation with two unknowns, the system response and backscattering amplitude of the target. The essence of using a standard target for calibration is that the scattering by the target can be accurately predicted, which normally requires an accurate scattering model and an accurate measure of the material properties and dimensions of the target. With this accurate knowledge, then the measured scattering by the target can be replaced by the predicted scattering:

$$
F_{\mathrm{bs}}^{(\mathrm{pred})}(\omega)=F_{\mathrm{bs}}^{(\mathrm{cal})}(\omega)
$$

Substituting this expression into Eq. (3) and rearranging terms gives an expression for the system response:

$$
H(\omega)=\frac{V_{r}^{(\mathrm{cal})}(\omega)}{V_{t}^{(\mathrm{cal})}(\omega) L^{(\mathrm{cal})}(\omega) F_{\mathrm{bs}}^{(\mathrm{pred})}(\omega)} .
$$

This is a general expression for the system response for both the full-wave and partial-wave calibration methods. For the full-wave case, the predicted scattering amplitude is simply 
the modal series solution to the scattering function where none of the various components of the scattering from the target are resolved. For narrowband systems this equation can be used directly in the calibration. However, for broadband systems, as discussed above, resolving the echo from the front interface can greatly facilitate the calibration. There are conditions under which the center frequency is high enough and the calibration sphere is large enough so that the raw, unprocessed echo from the front interface can be resolved and Eq. (5) can be used directly. However, for other cases when it cannot be resolved, the received signal needs to be processed so that the resolution approaches the theoretical limit, which is approximately equal to the inverse bandwidth of the system. In the next section, pulse compression processing is applied to the signal to improve the temporal resolution and Eq. (5) is expressed in terms of the processed signal.

\section{DETERMINING SYSTEM RESPONSE THROUGH PULSE COMPRESSION PROCESSING}

In order to resolve the echo from the front interface, pulse compression processing may need to be performed to improve the temporal resolution. A method similar to matched filter processing is used, which involves cross correlating the received signal with a "replica" signal (Chu and Stanton, 1998; Turin, 1960). Generally, a true replica would account for the system response. However, since it is the system response that is being determined, such a replica cannot be used. Furthermore, it is important that the replica match or come close to matching the echo from a single partial wave. If, in contrast, the replica fully accounted for all partial waves, then the compressed signal would resemble a sinc function with a single main lobe, with none of the partial waves resolved. By treating each of the partial waves as being due to a scattering center with a phase specific to its respective scattering process, then a replica chosen to match a single scattering center will tend to resolve the partial waves. For this purpose using the applied transmitter signal or one similar to it can be used as an approximation. Although use of these signals is not optimum, as they do not account for system response or perfectly match each partial wave, their use in pulse compression processing still significantly improves the temporal resolution toward the theoretical limit of the inverse bandwidth. Furthermore, another benefit of using a pulse-compression method (optimum or not) is that the signal-to-noise ratio significantly increases. Development and application of pulse compression techniques to scattering by marine organisms is described in Chu and Stanton (1998), including illustrations of treating each organism as a distributed target with different scattering centers.

Another issue concerns the need for enough separation between the echo from the front interface and subsequent echoes. With large enough separation, then the time "gate" or "window" used to process the echo from the front interface can be large enough to provide satisfactory spectral resolution on that echo for calculation of the system response. Having a sufficiently large separation also simultaneously satisfies the criterion of the temporal or "range" processing sidelobes of sequential echoes not overlapping, which other- wise would be a source of error. However, in a practical application, since the transducer response is nonuniform, the signal becomes temporally shaded, thus these sidelobes tend to be significantly reduced and are much less of a problem (Chu and Stanton, 1998). A detailed description of the gating process and associated spectral resolution is given in Sec. IV. Choice of diameter of sphere for a range of material properties and bandwidths that provides for a sufficiently long separation between the first and second echoes is described in Sec. V. The calculations given below associated with a partial wave analysis are based on the assumption of a sufficiently long separation.

The compressed pulse signal, $\mathrm{cp}_{1}(t)$, associated with the signal of interest, $v_{1}(t)$, is given by the following general expression:

$$
\mathrm{cp}_{1}(t) \equiv k_{\mathrm{cp}} v_{1}(t) \otimes v_{2}(t),
$$

where the normalization factor, $k_{\mathrm{cp}}$, is defined by

$$
k_{\mathrm{cp}} \equiv R_{2}^{-1}(0),
$$

where $R_{2}(t) \equiv v_{2}(t) \otimes v_{2}(t)$.

The term $\otimes$ denotes the correlation operation, and the signal $v_{2}(t)$ is either the replica (for matched filter processing) or a similar signal, such as the signal applied to the transmitter as discussed above.

As a result of this processing, the duration of $\mathrm{cp}_{1}(t)$ can be as short as (approximately) inverse bandwidth of the $v_{1}(t)$, regardless of the duration of $v_{1}(t)$. Thus, in the calibration experiment, for a sufficiently large bandwidth of the transmitter signal, it is possible that the desired partial waves (such as the echo from the front interface) can be resolved, even when those features are not resolved in the raw signal before processing.

Since it is desirable to determine the system response in the frequency domain, the Fourier transform of the compressed pulse signal given by Eq. (6) is first taken:

$$
\mathrm{CP}_{1}(\omega)=k_{\mathrm{cp}} V_{1}^{*}(\omega) V_{2}(\omega)
$$

where the superscript $*$ symbol denotes the complex conjugate.

Multiplying the numerator and denominator of Eq. (5) by the complex conjugate of the replica $V_{\text {rep }}^{(\mathrm{cal}) *}(\omega)$ and using Eq. (8) gives an expression for the system response in terms of the Fourier transforms of two compressed pulse signals:

$$
H(\omega)=\frac{\mathrm{CP}_{r}^{(\mathrm{cal})^{*}}(\omega)}{L^{\text {(cal) }}(\omega) C P_{\text {pred }}^{(\text {conn)* }}(\omega)},
$$

where $\mathrm{CP}_{r}^{(\mathrm{cal})}(\omega)$ and $C P_{\text {pred }}^{\text {(conv) }}(\omega)$ are the Fourier transforms of the compressed pulse signals, $\mathrm{cp}_{r}^{(\text {(cal })}(t)$ and $\mathrm{cp}_{\text {pred }}^{\text {(conv) }}(t)$, respectively, as defined below:

$$
\mathrm{cp}_{r}^{(\mathrm{cal})}(t) \equiv k_{\mathrm{cp}}^{(\mathrm{cal})} v_{r}^{(\mathrm{cal})}(t) \otimes v_{\text {rep }}^{(\mathrm{cal})}(t)
$$

and

$$
\mathrm{cp}_{\text {pred }}^{\text {(conv) }}(t) \equiv k_{\mathrm{cp}}^{(\mathrm{cal})}\left\{f_{\mathrm{bs}}^{(\mathrm{pred})}(t) * v_{t}^{(\mathrm{cal})}(t)\right\} \otimes v_{\text {rep }}^{(\text {cal })}(t) .
$$

Here, $\mathrm{cp}_{r}^{(\mathrm{cal})}(t)$ is the compressed pulse associated with the calibration measurement in which the received voltage due 
to the backscattering by the standard target is cross correlated with the replica signal, $v_{\text {rep }}^{(\text {cal })}(t)$. Again, as stated above, this does not need to be a true replica. The second term, $\mathrm{cp}_{\mathrm{pred}}^{\text {(conv) }}(t)$, is more complex. First, the impulse response $f_{\mathrm{bs}}^{\text {pred }}(t)$ of the backscattering amplitude of the standard target is predicted through calculating the inverse Fourier transform of the modal series solution and convolved $(*)$ with the voltage signal $v_{t}^{\text {(cal) }}(t)$ applied to the transmitting transducer in the calibration measurement (here, the convolution operator, ${ }^{*}$, appears as a product-like operator, not a superscript). This convolution product is then cross correlated with the replica signal, which results in the compressed pulse signal. The normalization constant, $k_{\mathrm{cp}}^{(\mathrm{cal})}$, which is defined in Eq. (7) [where $\left.v_{2}(t)=v_{\text {rep }}^{\text {(cal) }}(t)\right]$, is the same for each compressed pulse signal in Eq. (9) and cancels out (implicitly) as it appears as a factor to both the numerator and denominator. The derivation of Eq. (9) makes use of the fact that the product $F_{\mathrm{bs}}^{(\text {pred })}(\omega) V_{t}^{(\text {cal })}(\omega)$ in the frequency domain is the Fourier transform of the convolution product $f_{\mathrm{bs}}^{\text {(pred) }}(t) * v_{t}^{(\text {cal })}(t)$ in the time domain.

Equation (9) is a general equation that can be used for either a full-wave analysis or partial-wave analysis. That is, once the measured signal of interest is compressed through the processing, the time gate can be chosen to either include just one partial wave (such as the echo from the front interface of the sphere) or all partial waves. Given the generality, it is important that the same time gate is used in both the calibration measurement and the predictions. Specifically, if a particular partial wave is selected in the calibration measurement to give $\mathrm{cp}_{r}^{(\mathrm{cal})}(t)$, then that same corresponding wave must be selected in the impulse response, $f_{\mathrm{bs}}^{(\text {pred })}(t)$.

\section{AT-SEA CALIBRATION OF BROADBAND SYSTEM}

The above formulation is applied to an experiment at sea in which a broadband system was calibrated. The calibrations took place near Provincetown, MA (tip of Cape Cod) in 50-m-deep water using the R/V Tioga on September 11, 2006. The system, made by Edgetech, consisted of three broadband transducers, including a Reson transducer (Model TC2138), which is the subject of this study (Stanton et al., 2007). The Reson transducer was sensitive over a band of approximately $30-105 \mathrm{kHz}$ and a linear frequency modulated signal ("chirp") was applied to that transducer (Fig. 2). This range is not the bandwidth as conventionally defined relative to the $-3 \mathrm{~dB}$ points of the spectrum but the range over which the system has usable energy.

The echoes were digitized at a sampling rate of $260 \mathrm{kHz}$ and processed at that same rate without downsampling. The chirp signal was chosen as it is a commonly used signal and it provided the necessary resolution in this experiment, although it is not necessarily the optimal signal. As a result of pulse-compression processing, the original $2 \mathrm{~ms}$ pulse (which corresponds to a range resolution of $1.5 \mathrm{~m}$ ) is compressed to a signal with a duration of about $35 \mu$ s (which corresponds to a range resolution of $3 \mathrm{~cm}$ ). This value of $35 \mu \mathrm{s}$ is greater than the ideal value of $11.4 \mu$ s shown in
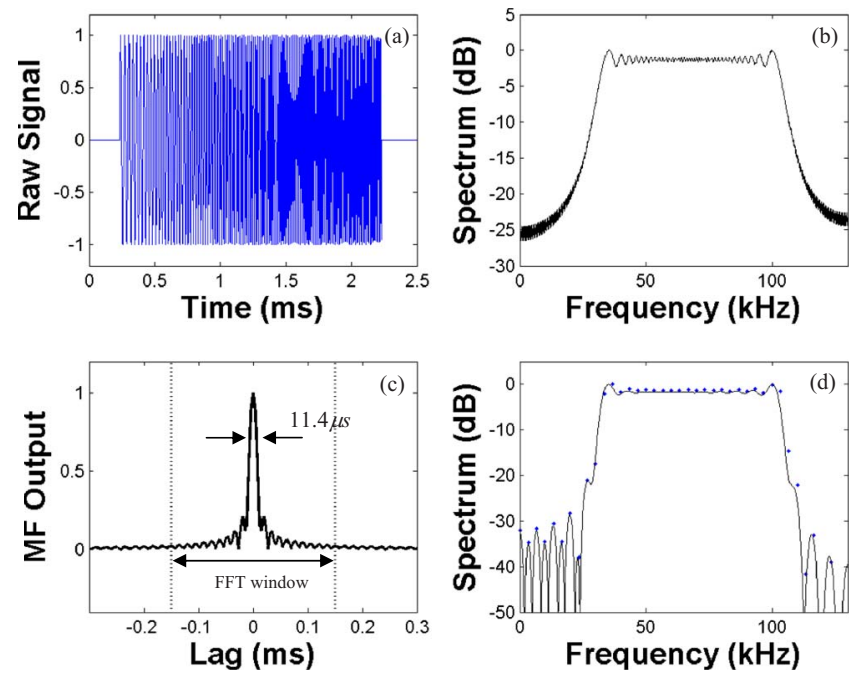

FIG. 2. (Color online) Effects of pulse-compression processing of a broadband signal. (a) A 2-ms-long (raw) chirp signal applied to Reson transducer, (b) frequency spectrum of signal from (a), (c) envelope of pulse-compressed signal in which autocorrelation function of signal shown in (a) is calculated, (d) frequency spectrum of pulse-compressed signal (before envelope was calculated) from (c). Dots: Spectrum calculated using 300- $\mu$ s-long window shown in (c). Solid: spectrum calculated using 10-ms-long window, which involves beginning with the signal within the $300-\mu$ s-long window and zero padding each side.

Fig. 2 due to a combination of factors: the transducer response is not uniform over the band and a true replica was not used in the processing.

The system, which is normally towed in surveys with its acoustic beams aimed downward, was suspended $10 \mathrm{~m}$ directly below the vessel, which was anchored. The system was tethered so that the acoustic beams would still be aimed generally in the downward direction. Several calibration spheres were used, one at a time, suspended $30 \mathrm{~m}$ below the towbody. For the 41.5-cm-diam air-filled spherical aluminum shell discussed in this paper $(4.1 \mathrm{~mm}$ average wall thickness), a $110 \mathrm{lb}$ weight was tethered with a 5-m-long line to the bottom of the sphere since the sphere was positively buoyant by approximately $95 \mathrm{lbs}$.

The towbody, sphere, and weight were all free to move, which caused significant variability in the echo level which needed to be accounted for. The most significant movement was the pitch and roll of the towbody due to the up and down motion of the vessel in the waves. The pitch and roll angles were sampled at a high rate relative to the motion. In plotting echo amplitude versus pitch and roll angle (not shown) the highest amplitude echoes were tightly clustered around a value near pitch $=$ roll $=0^{\circ}$. These are assumed to be the echoes from very close to or at the center of the acoustic beam and are the ones analyzed in this paper. Although this was not an ideal approach toward determining the location of the target in the beam, it was satisfactory for the purpose of this study. Use of an acoustic imaging system, such as a multibeam, split beam, or lens system, outside of the band of interest and used simultaneously, could have directly provided the information. Or, had the broadband system itself been an imaging system, it could have directly provided that information as well. 
The replica signal used in the pulse-compression processing was based on a separate experiment conducted in a laboratory tank in which the system was aimed upward at the smooth air-water interface. The echo from the interface was used as the replica signal in the at-sea calibration. Since the echo from the interface was so strong, and to prevent saturation, the transmission power in the laboratory measurement was significantly reduced resulting in significant distortion in the spectrum of the echo. The distortion is presumably due to nonlinearities in the transmission/reception isolation electronics which operate on the assumption of a strong transmission signal. Because of this nonlinearity, the laboratory echo is not a true replica. In spite of the fact that the signal was not a true replica, it still significantly improved the resolution of the calibration echoes through pulse-compression processing. This particular waveform was used because of its availability. As discussed earlier, and for practical reasons, other waveforms such as the signal applied to the transmitter could have been used as well, providing the desired improvement in resolution.

Another issue involves the fact that a gate of finite duration was used in the analysis. The gate needed to be small enough to adequately resolve the echo from the front interface, but not so small so as to compromise the spectral resolution of the measurement. A gate of $300 \mu$ s was used which resolved the echo from the front interface and provided a spectral resolution of $3.3 \mathrm{kHz}$. Effects of different gate durations on the calculated spectrum of the compressed pulse are shown in Fig. 2. Zero padding values of the signal before and after the window are shown to create a finer spectral grid, although the inherent resolution is not improved beyond the value of the inverse gate duration.

Once the echoes in the at-sea calibration were temporally compressed through pulse-compression processing, the various scattering features from the sphere were resolved (top panel of Fig. 3; envelope of processed signal). The envelope of the compressed-pulse signal shows a strong initial return which corresponds to the echo from the front interface. At approximately $0.6 \mathrm{~ms}$ after that first echo is another strong echo associated with a circumferential wave. The impulse response of the exact solution for the scattering amplitude gives qualitatively similar results in that there is a strong initial echo and a strong secondary echo at about $0.6 \mathrm{~ms}$ later (middle panel of Fig. 3). In addition, both the data and the impulse response show small echoes between the two much stronger echoes. Part of the calibration formulation involves convolving the applied transmit signal with the impulse response. This convolution process broadens the echoes in the impulse response (bottom panel of Fig. 3; the envelope of the convolved signal is illustrated). Also, calculation of the impulse response involves an inverse Fourier transform over a finite range of frequencies. Artifacts of truncating the spectrum were suppressed through use of an amplitude weighting function.

The spectrum of the compressed-pulse echo from the calibration sphere was calculated using the data in the top panel of Fig. 3 over two different time gates; a short, $300-\mu$ s-long gate including only the echo from the front interface (with that echo centered in the gate) and a longer gate
Air-filled Aluminum Shell
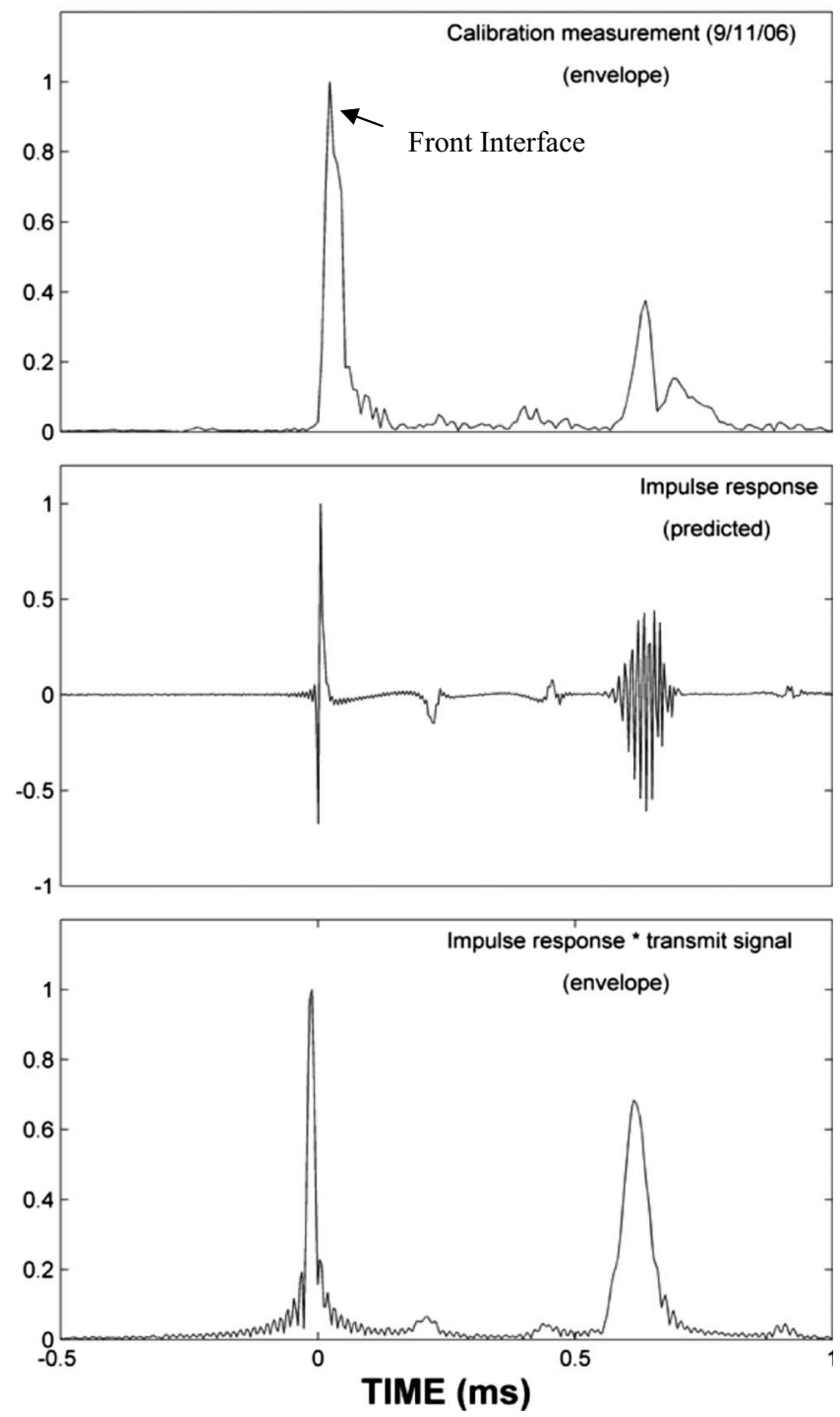

FIG. 3. Time-domain analysis of calibration signal. Top panel: Envelope of pulse-compressed echo from air-filled aluminum spherical shell in at-sea measurement. The echo was cross correlated with echo measured in a tank experiment with the system aimed up at the smooth air-water interface. Middle panel: Impulse response of predicted echo from spherical shell through calculating inverse Fourier transform of exact modal series solution. Bottom panel: Envelope of the convolution of the impulse response from middle panel and the applied transmitter signal. Each plot is normalized to unity. The data were collected on September 11, 2006 with the 41.5-cmdiam sphere suspended $30 \mathrm{~m}$ below the towbody.

( $3 \mathrm{~ms}$ ) that includes the entire series of partial wave echoes. Once gated, each signal was "zero padded" to form a 4-mslong signal for Fourier analysis. The results illustrate the significant differences in spectra of the different components of the echo [Fig. 4(a)]. The spectrum of the echo from the front interface alone is smoothly varying over the entire band while the spectrum of the entire echo (i.e., front interface and circumferential waves), is rapidly oscillating over the band. This observation is consistent with the theoretical predictions discussed in Sec. I where the scattering amplitude of the echo from the front interface alone (i.e., partial wave) is weakly dependent upon frequency and the scattering amplitude from all echoes from the sphere (i.e., full wave) is 

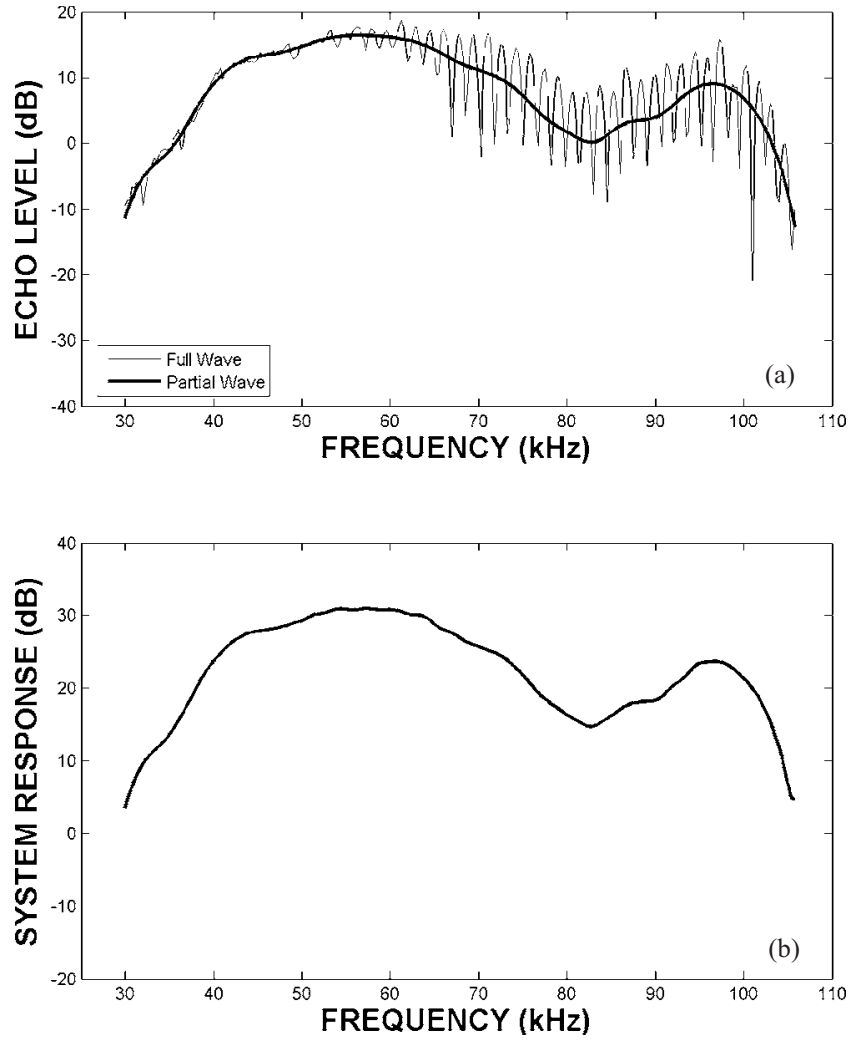

FIG. 4. Spectra of signals and system response associated with calibration measurement with air-filled spherical shell. Upper panel: Spectrum of (partial wave) echo from front interface (thick line) compared with spectrum of (full wave) echo from all partial waves (thin line). The spectra were calculated using the pulse-compressed signal illustrated in the top panel of Fig. 3 . Lower panel: System response of broadband transducer as derived through partial-wave analysis using echo from front interface.

strongly dependent upon frequency due to the frequencydependent interference between the various partial waves.

Using the equations described in Sec. III, the system response is determined through the partial-wave analysis [Fig. 4(b)]. Here, the measured echo from the front interface (top panel of Fig. 3) is used in combination with the corresponding echo in the predicted impulse response convolved with the signal applied to the transmitter (bottom panel of Fig. 3) to produce the system response. As with Fig. 4(a), the echo from the front interface was obtained through a $300-\mu$ s-long gate, zero padded to $4 \mathrm{~ms}$ long. This response is shown to be quite similar to the spectrum of the pulsecompressed echo of the front interface [Fig. 4(a)]. The similarity is due to the fact that the scattering amplitude of that partial wave depends weakly upon frequency, resulting in the majority of the frequency dependence of that echo being due to the system response.

The partial-wave-based response shown in Fig. 4(b) has also been compared with the response determined by a fullwave analysis from data involving a smaller $(20 \mathrm{~cm}$ diameter) solid aluminum sphere where there are fewer resonances (not shown). Although the overall levels and trends of the two results were quite similar, there were significant differences at resonance frequencies where the full-wave solution is prone to error. An attempt was made to reduce the errors in the full-wave analysis by smoothing the calculated
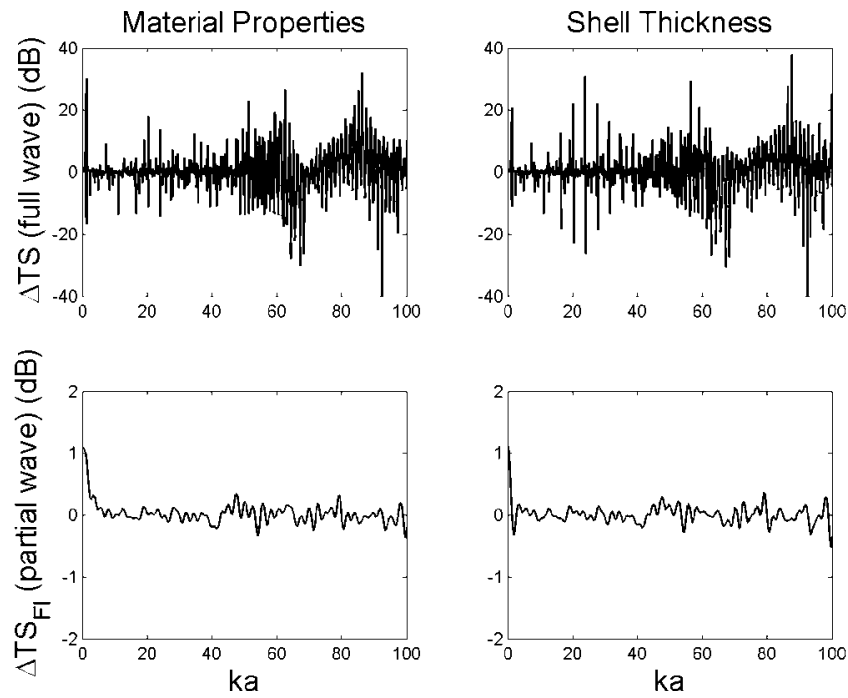

FIG. 5. Sensitivity analysis of full-wave (upper panels) and partial-wave (lower panels) echoes for aluminum spherical shell, whose shell thickness is about $1 \%$ of the diameter. Material properties (density, compressional sound speed, and shear sound speed) of spherical shell are varied by about $1 \%$ between two sets of predictions in the left panels. Shell thickness is varied by $7 \%$ between two sets of predictions in the right panels.

response. Even after the smoothing, there were errors in the full-wave analysis of up to $3 \mathrm{~dB}$ due to the uncertainties associated with the nulls.

\section{DESIGNING A CALIBRATION SPHERE AND SIGNAL PROCESSING APPROACH}

Choice of the sphere and signal processing approach depends strongly upon the center frequency and bandwidth of the acoustic signal. For a narrowband system, the previously published methods cited above can be used which involve choosing the diameter and material properties of the sphere so that the center frequency does not involve a resonance of the sphere. Specifically, the frequency of the acoustic system is in a section of the curve illustrating scattering amplitude versus frequency that is relatively insensitive to frequency and a full-wave analysis is performed. However, as discussed above, once the bandwidth of the system spans multiple resonances, then a partial wave analysis should be considered in order to eliminate the sensitivity of the calibration to the resonances. These design criteria are elaborated upon below.

\section{A. Sensitivities of partial-wave and full-wave analysis with respect to uncertainties in material properties and shell thickness}

A calibration involving a partial-wave analysis which makes use of the echo from the front interface of the sphere is relatively insensitive to changes in material properties and, in the case of spherical shells, the thickness of the shell (Fig. 5). This insensitivity is due to the fact that the echo from the interface does not contain any resonances, as shown in Eq. (1) and Fig. 1(a). However, if a full wave analysis were to be applied to a broadband system in which the spectrum of the signal spanned many resonances, the calibration would be extremely sensitive to changes in material properties and 


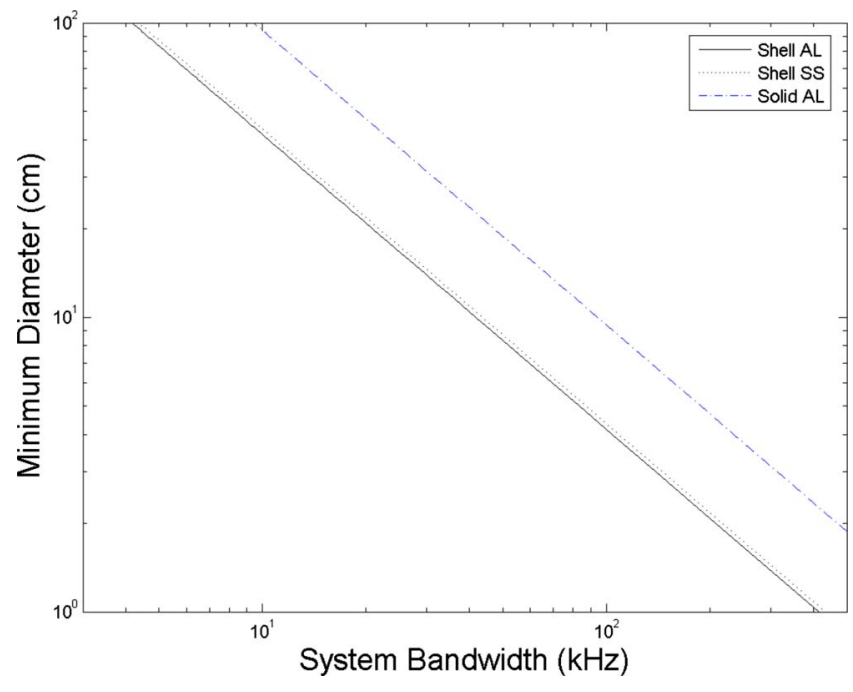

FIG. 6. (Color online) Design curves for minimum diameter of calibration sphere that can be used in partial-wave analysis. Combinations of solid elastic and air-filled elastic shell for aluminum ("AL") and stainless steel ("SS") are given. A separation between the echo from the front interface and the next major partial wave is assumed to be two times the inherent temporal resolution of the system (i.e., two times the inverse bandwidth). A larger than minimum diameter will allow for a longer processing gate and corresponding improved spectral resolution.

thickness of shell. Since the resonances are very narrow, then it only takes a slight change in those parameters to shift the resonance an amount comparable to its width. Since the resonance gives rise to variations in scattering amplitude of up to $10-20 \mathrm{~dB}$, and since the calibration involves dividing by the predicted scattering amplitude, then a slight shift in resonance due to uncertainties in the properties can cause errors near those frequencies of up to $10-20 \mathrm{~dB}$ (Fig. 5). Thus, it is clear that in order to avoid such strong sensitivities, a partialwave analysis should be considered.

\section{B. Diameter of sphere}

A partial-wave analysis can be used only when the echo from the front interface is resolved from the other echoes from the sphere. The resolution requirement is a function of diameter of the sphere, bandwidth of the system, frequencies involved, material properties, and whether or not the sphere is a solid or shell. The combination of material properties, type of sphere (solid or shell), diameter, and frequencies involved will determine the speed of the circumferential waves. Knowledge of the speed of those waves (especially the speed of the first arrival after the echo from the front interface), in combination with the bandwidth of the system, will dictate the smallest diameter of sphere to use. Given the complexity of this problem, the diameter should be determined through simulations of the scattering. At sufficiently high values of $k a$ and for fixed material properties and type of sphere, the minimum diameter of sphere to be used in the calibration varies inversely with bandwidth (Fig. 6). In addition, our simulations show that the minimum diameter depends strongly upon whether or not the sphere is solid or an air-filled thin-walled spherical shell. In our particular case of spherical shells, the minimum diameter does not depend strongly upon material type (e.g., aluminum vs. stainless steel) (Fig. 6).

\section{SUMMARY AND CONCLUSIONS}

An approach has been developed for calibrating broadband active acoustic systems with a single standard spherical target. The approach involves a partial-wave analysis which uses the echo from the front interface of a calibration sphere. This echo is relatively insensitive to acoustic frequency, in contrast to the full-wave signal that contains significant narrow resonances. Furthermore, the echo is relatively insensitive to uncertainties in material properties and sphere dimensions. Isolating this echo for analysis requires resolving it from the subsequent echoes, such as circumferential waves. Pulse-compression processing was incorporated into the approach, resulting in significantly improved temporal resolution. The calibration equations were written in terms of the pulse-compressed echoes. Also, design curves were presented for the recommended minimum diameter of the sphere as a function of system bandwidth and material properties of the sphere.

This partial-wave analysis is an attractive alternative to the traditional full-wave analysis for the calibration of broadband systems. As a result of the complete elimination of the source of resonances, this approach significantly reduces errors in the calibration associated with uncertainties or errors in values of material properties and sphere dimensions. Furthermore, through use of pulse-compression processing, the diameter of the sphere used in the calibration can be minimized.

\section{ACKNOWLEDGMENTS}

The authors are grateful to the following people from the Woods Hole Oceanographic Institution (WHOI), Woods Hole, MA, for their assistance on this project: Ken Houtler (captain of the R/V Tioga), Jeff Lord, Don Peters, and Shirley Barkley. This work was supported by the U.S. Office of Naval Research Grant Nos. N00014-04-1-0475 and N0001404-1-0440 and the J. Seward Johnson Chair at WHOI.

Chu, D., and Stanton, T. K. (1998). "Application of pulse compression techniques to broadband acoustic scattering by live individual zooplankton," J. Acoust. Soc. Am. 104, 39-55.

Dragonette, L. R., Numrich, S. K., and Frank, L. J. (1981). "Calibration technique for acoustic scattering measurements," J. Acoust. Soc. Am. 69, 1186-1189.

Feuillade, C., Meredith, R. W., Chotiros, N. P., and Clay, C. S. (2002). "Time domain investigation of transceiver functions using a known reference target," J. Acoust. Soc. Am. 112, 2702-2712.

Foote, K. G. (1982). "Optimizing copper spheres for precision calibration of hydroacoustic equipment," J. Acoust. Soc. Am. 71, 742-747.

Foote, K. G. (2006). "Optimizing two targets for calibrating a broadband multibeam sonar," Proc. Oceans 2006 MTS/IEEE, (Boston).

Foote, K. G., Atkins, P. R., Bongiovanni, C. C., Francis, D. T. I., Eriksen, P. K., Larsen, M., and Mortensen, T. (1999). "Measuring the frequency response function of a seven-octave bandwidth echo sounder," Proc. I.O.A., Vol. 21(1), pp. 88-95.

Foote, K. G., Francis, D. T. I., and Atkins, P. R. (2007). "Calibration sphere for low-frequency parametric sonars," J. Acoust. Soc. Am. 121, 14821490.

Hackman, R. H. (1993). "Acoustic scattering from elastic solids," in Physical Acoustics, edited by A. D. Pierce and R. N. Thurston (Academic, San Diego) Vol. XXII, Chap. 1, pp. 1-194. 
Kaduchak, G., Kwiatkowski, C. S., and Martson, P. L. (1995). "Measurement and interpretation of the impulse response for backscattering by a thin spherical shell using a broad-bandwidth source that is nearly acoustically transparent," J. Acoust. Soc. Am. 97, 2699-2708.

Marston, P. L. (1992). "Geometrical and catastrophe optics methods in scattering," in Physical Acoustics, edited by A. D. Pierce and R. N. Thurston (Academic, San Diego), Vol. XXI, Chap. 1, pp. 2-234.

Numrich, S. K., and Überall, H. (1992). "Scattering of sound pulses and the ringing of target resonances," in Physical Acoustics, edited by A. D. Pierce and R. N. Thurston (Academic, San Diego), Vol. XXI, Chap. 2, pp. 235 318.
Simmonds, J., and MacLennan, D. (2005). Fisheries Acoustics: Theory and Practice, 2nd ed. (Blackwell, Oxford, UK).

Stanton, T. K. (1990). "Sound scattering by spherical and elongated shelled bodies," J. Acoust. Soc. Am. 88, 1619-1633.

Stanton, T. K., Chu, D., Jech, J. M., and Irish, J. D. (2007). "A broadband echosounder for resonance classification of swimbladder-bearing fish," Proc. Oceans 2007 IEEE (Aberdeen).

Sun, Z., Gimenez, G., Vray, D., and Denis, F. (1991). "Calculation of the impulse response of a rigid sphere using the physical optic method and modal method jointly," J. Acoust. Soc. Am. 89, 10-18.

Turin, G. L. (1960). "An introduction to matched filters," IRE Trans. Inf. Theory IT-6, 311-329. 